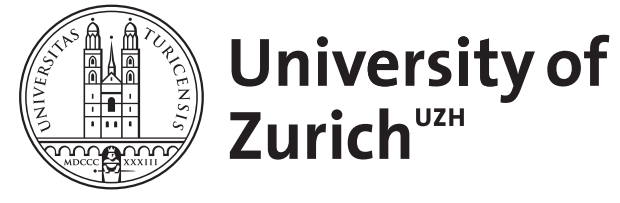
Archive

University of Zurich

University Library

Strickhofstrasse 39

CH-8057 Zurich

www.zora.uzh.ch

Year: 2015

Genetische Formen der distalen renal-tubulären Azidose

Mohebbi, Nilufar ; Wagner, Carsten A

DOI: https://doi.org/10.1007/s11560-014-0946-1

Posted at the Zurich Open Repository and Archive, University of Zurich

ZORA URL: https://doi.org/10.5167/uzh-122755

Journal Article

Accepted Version

Originally published at:

Mohebbi, Nilufar; Wagner, Carsten A (2015). Genetische Formen der distalen renal-tubulären Azidose.

Der Nephrologe, 10:214-222.

DOI: https://doi.org/10.1007/s11560-014-0946-1 


\begin{tabular}{|l|l|}
\hline DOI & $10.1007 / \mathrm{s} 11560-014-0946-1$ \\
\hline Copyright & Springer-Verlag Berlin Heidelberg - 2015 \\
\hline
\end{tabular}

\title{
Leitthema
}

\section{Genetische Formen der distalen renal-tubulären Azidose}

\section{Besonderheiten im Erwachsenenalter}

\author{
N. Mohebbi ${ }^{1}$ - C.A. Wagner ${ }^{2}$ \\ ${ }^{1}$ Klinik für Nephrologie, Universitätsspital Zürich, Zürich, Schweiz \\ ${ }^{2}$ Physiologisches Institut, Universität Zürich, Zürich, Schweiz
}

\section{Korrespondenzadresse}

PD Dr. N. Mohebbi

Klinik für Nephrologie, Universitätsspital Zürich

Rämistr. 100

$\mathrm{CH}-8091$ Zürich

Schweiz

nilufar.mohebbi@usz.ch

\section{PD Dr. N. Mohebbi}

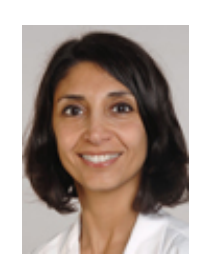

Online publiziert: Onlinedatum erscheint nach Freigabe

\section{Zusammenfassung}

Hintergrund Die distale renal-tubuläre Azidose (dRTA) ist eine tubuläre Erkrankung mit einem primären Defekt in den Sammelrohren, der zu einer inadäquaten Ausscheidung von Protonen, Ammonium und titrierbaren Säuren führt. Die Folge ist eine hyperchlorämische 
metabolische Azidose mit einem alkalischen Urin-pH. Bisher sind insgesamt 3 Gene (SLC4A1, ATP6V1B1, ATP6V0A4) beschrieben, welche eine dRTA verursachen können. SLC4A1-Mutationen werden meist autosomal-dominant, in wenigen Fällen aber auch autosomal-rezessiv vererbt. Die Erkrankung kann im Kindesalter und auch im jungen Erwachsenenalter auftreten. ATP6V1B1- und ATP6V0A4-Mutationen betreffen jeweils 2 Untereinheiten (B1- und a4-Untereinheit) der $\mathrm{H}^{+}$-ATPase-Protonenpumpe und werden autosomal-rezessiv vererbt. Bei beiden Mutationen tritt die Erkrankung typischerweise im frühen Kindesalter auf. Heterozygote Träger einer ATP6V1B1-oder ATP6V0A4-Mutation haben vermutlich ein erhöhtes Risiko für eine Nephrolithiasis bzw. Nephrokalzinose.

Ergebnisse Die klinischen Symptome können sich mild in Form von leichter metabolischer Azidose und Nierensteinen oder eher ausgeprägt in Form von Gedeihstörung, schwerer metabolischer Azidose und Nephrokalzinose bis hin zur Niereninsuffizienz präsentieren. Bei der autosomal-rezessiven Form (ATP6V1B1- oder ATP6V0A4-Mutationen) tritt bei einer Mehrzahl der Patienten zusätzlich eine fortschreitende Innenohrschwerhörigkeit auf, teilweise kombiniert mit einem erweiterten vestibulären Aquäduktsyndrom.

Schlussfolgerung Die dRTA ist eine wichtige Differenzialdiagnose bei Kindern, aber auch bei jungen Erwachsenen mit Nephrokalzinose oder rezidivierenden Nierensteinleiden, insbesondere im Falle von 100\%igen Apatitsteinen oder gleichzeitiger Schwerhörigkeit, die eine medizinische Versorgung mit Hörhilfen erfordert.

\section{Schlüsselwörter}

Genmutationen $\cdot$ Urinansäuerung $\cdot$ Nephrokalzinose $\cdot$ Nephrolithiasis $\cdot$ Sensorineurale Innenohrschwerhörigkeit

\section{Inherited forms of distal renal tubular acidosis}

\section{Characteristics in adulthood}

\section{Abstract}

Background Distal renal tubular acidosis (dRTA) is characterized by defective urinary acidification resulting in impaired excretion of protons, ammonium and titratable acids. Subsequently, patients develop hyperchloremic metabolic acidosis with an inappropriately alkaline urine. Inherited forms of dRTA are caused by mutations in three different genes, namely SLC4A1, ATP6V1B1 and ATP6VOA4 where SLC4A1 mutations mostly occur in 
an autosomal dominant manner and rarely as recessive mutations. The ATP6V1B1 and ATP6V0A4 mutations each affect two different subunits of the vacuolar $\mathrm{H}^{+}$-ATPase proton pump, the B1 and a4 subunits and are inherited in an autosomal recessive manner. Clinical manifestations of dRTA usually occur during infancy or childhood. Heterozygous carriers of ATP6V1B1 and ATP6VOA4 mutations may have a higher risk to develop nephrolithiasis and nephrocalcinosis, respectively.

Results Depending on the respective gene mutation patients may present with mild clinical symptoms, such as mild metabolic acidosis and incidental detection of kidney stones or severe manifestations, such as failure to thrive, severe metabolic acidosis and nephrocalcinosis. Progressive sensorineural hearing loss develops in the majority of patients with recessive dRTA (ATP6V1B1 and ATP6V0A4 mutations). Some patients with recessive dRTA may also develop abnormal widening of the vestibular aqueduct.

Conclusion Distal RTA is an important differential diagnosis in children as well as in young adults with nephrocalcinosis or recurrent kidney stone formation, particularly if stone composition consists of $100 \%$ apatite or if there is history of hearing abnormalities.

\section{Keywords}

Gene mutations $\cdot$ Urinary acidification $\cdot$ Nephrocalcinosis $\cdot$ Nephrolithiasis $\cdot$ Sensorineural hearing loss

Die Niere spielt bei der Regulation und Aufrechterhaltung des Säure-Basen-Haushalts eine wichtige Rolle. Dabei ist insbesondere die tägliche renale Ausscheidung bzw. Pufferung der durch den Metabolismus generierten Säureäquivalente von etwa $1 \mathrm{mmol}$ pro Kilogramm Körpergewicht essenziell. Die Bedeutung dieser Aufgabe zeigt sich vor allem durch die verschiedenen Formen von renalen Azidosen, die bei angeborenen oder erworbenen Störungen der renalen Säureausscheidung sowie bei fortgeschrittener Niereninsuffizienz auftreten können. 


\section{Physiologische Grundlagen der renalen Säureausscheidung}

Die Aufrechterhaltung des Säure-Basen-Haushalts durch die Niere beinhaltet 3 wichtige Prozesse:

- Rückresorption von filtriertem Bikarbonat im proximalen Tubulus,

- De-novo-Synthese von Bikarbonat während der Ammoniagenese im proximalen Tubulus,

- Ausscheidung von Protonen und Ammonium entlang des Sammelrohrs [1].

Die Nieren filtrieren täglich etwa 1801 Primärharn, in dem unter anderem rund $4500 \mathrm{mEq}$ Bikarbonat enthalten sind, welches bei gesunden Erwachsenen komplett aus dem Primärharn rückresorbiert wird. Dabei wird der größte Anteil des Bikarbonats entlang des proximalen Tubulus durch die gleichzeitige Sekretion von Protonen in den Primärharn mittels Natrium-Protonen-Austauscher (NHE, v. a. die Isoform NHE3/SLC9A3) und Protonenpumpen ( $\mathrm{H}^{+}$-ATPasen) initiiert. Durch die Anwesenheit von Karboanhydrasen [v. a. Karboanhydrase Typ IV (CAIV)] kommt es zur Bildung von $\mathrm{CO}_{2}\left(\right.$ aus $\left.\mathrm{H}^{+}+\mathrm{HCO}_{3}^{-}\right)$im Urin, welches in die proximalen Tubuluszellen diffundiert, wo wiederum unter dem Einfluss von Karboanhydrasen [v. a. Karboanhydrase Typ II (CAII)] $\mathrm{H}^{+}$und $\mathrm{HCO}_{3}{ }^{-}$gebildet werden. Das somit rückresorbierte Bikarbonat kann durch den Natrium-Bikarbonat-Kotransporter (NBCe1/SLC4A4) in das Blut abgeben werden. Die Protonen werden wiederum in das Lumen sezerniert (Abb. 1). Ein geringer Anteil des filtrierten Bikarbonats wird in einem späteren Abschnitt des Tubulusapparats entlang des aufsteigenden Teils der dicken Henle-Schleife und im distalen konvoluten Tubulus rückresorbiert. Die Mechanismen ähneln hier dem proximalen Tubulus. Ein weiterer sehr geringer Teil des Bikarbonats wird entlang des proximalen Tubulus parazellulär und somit ATP-unabhängig rückresorbiert. Hierbei spielt vor allem die hohe luminale Chloridkonzentration eine wichtige Rolle, die zu einer negativen Ladung des Lumens führt, welche wiederum die parazelluläre Bikarbonatrückresorption begünstigt. Des Weiteren begünstigt der parazelluläre Transport von $\mathrm{H}_{2} \mathrm{O}$ und weiteren Ionen (durch den sog. „Solvent-drag“-Mechanismus) an dieser Stelle ebenfalls die parazelluläre Bikarbonatresorption. 


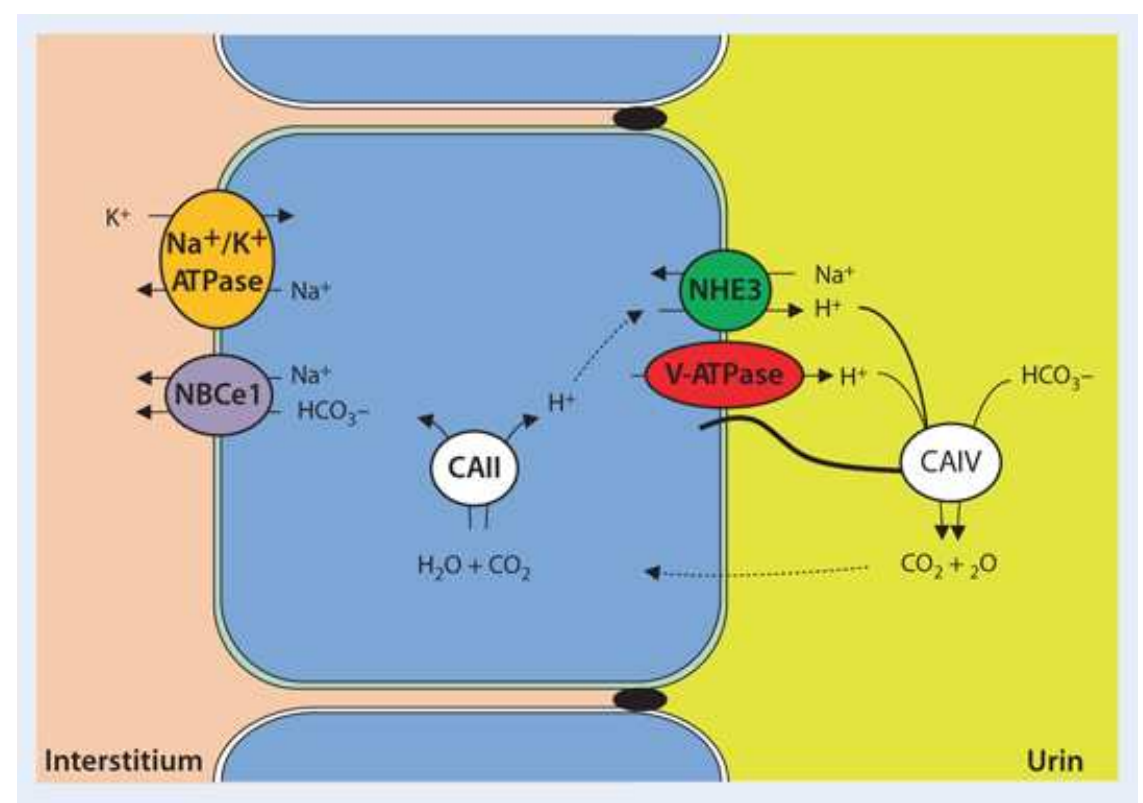

Abb. 1 Bikarbonatrückresoprtion im proximalen Tubulus (NBCe1 Natrium-Bikarbonat-Kotransporter, NHE3 Natrium-Protonen-Austauscher 3, V-ATPase Vakuolen-ATPase, CAII Karboanhydrase Typ II, CAIV Karboanhydrase Typ IV)

Die durch den Stoffwechsel produzierte Säure muss unter Verbrauch von Bikarbonat gepuffert werden. Das dabei verbrauchte Bikarbonat wird mehrheitlich durch eine renale De-novo-Synthese im proximalen Tubulus und im Sammelrohr im Rahmen der Ammoniogenese wieder bereitgestellt, wobei aus dem Abbau von 1 Glutamin 2 Ammoniak $\left(\mathrm{NH}_{3}\right)$ - und 2 Bikarbonatmoleküle gebildet werden. Zusätzlich werden 2 weitere Bikarbonatmoleküle durch die Pufferung von Protonen und die Bildung von Ammonium $\left(\mathrm{NH}_{4}^{+}\right)$gewonnen. Dieser Prozess ist an die Glukoneogenese gekoppelt, sodass hier u. a. Insulin eine regulatorische Rolle einnehmen kann. Bei vermehrter Säurebelastung (endogen oder exogen) werden die Ammoniogenese und die damit verbundene renale Ammoniumausscheidung massiv gesteigert und stellen somit einen der wichtigsten Adaptationsprozesse zur Regulation des Säure-Basen-Status dar. Durch die parallel gesteigerte Aufnahme von Glutamin und die Hochregulation der Enzyme der Ammoniogenese wird das neu gebildete Ammoniak zum größten Teil im proximalen Tubulus in den Urin abgegeben und nach Bindung an ein Proton und Bildung von Ammonium $\left(\mathrm{NH}_{4}^{+}\right)$ in der Henle-Schleife größtenteils rückresorbiert, im Nierenmark angereichert und entlang des Sammelrohrs endgültig in den Urin sezerniert. 
wichtigen Adaptationsprozesse zur Regulation

des Säure-Basen-Status dar

In den Typ-A-Schaltzellen (auch als Zwischenzellen bekannt) des Sammelrohrs besteht ebenfalls die Möglichkeit der Bikarbonatneubildung, indem die aus $\mathrm{CO}_{2}$ und $\mathrm{H}_{2} \mathrm{O}$ entstandene Kohlensäure $\left(\mathrm{H}_{2} \mathrm{CO}_{3}\right)$ mit Hilfe von Karboanhydrase II zu Protonen und Bikarbonat umgewandelt wird und das neu gebildete Bikarbonat über einen basolateralen Chlorid-Bikarbonat-Austauscher [AE (,anion exchanger“)1/SLC4A1] an das Blut abgegeben wird (Abb. 2; $[\underline{2}, \underline{3}])$. Seltene genetische Defekte in diesem Austauscher sind Ursache für angeborene Formen einer distalen renal-tubulären Azidose (dRTA; [4]]).

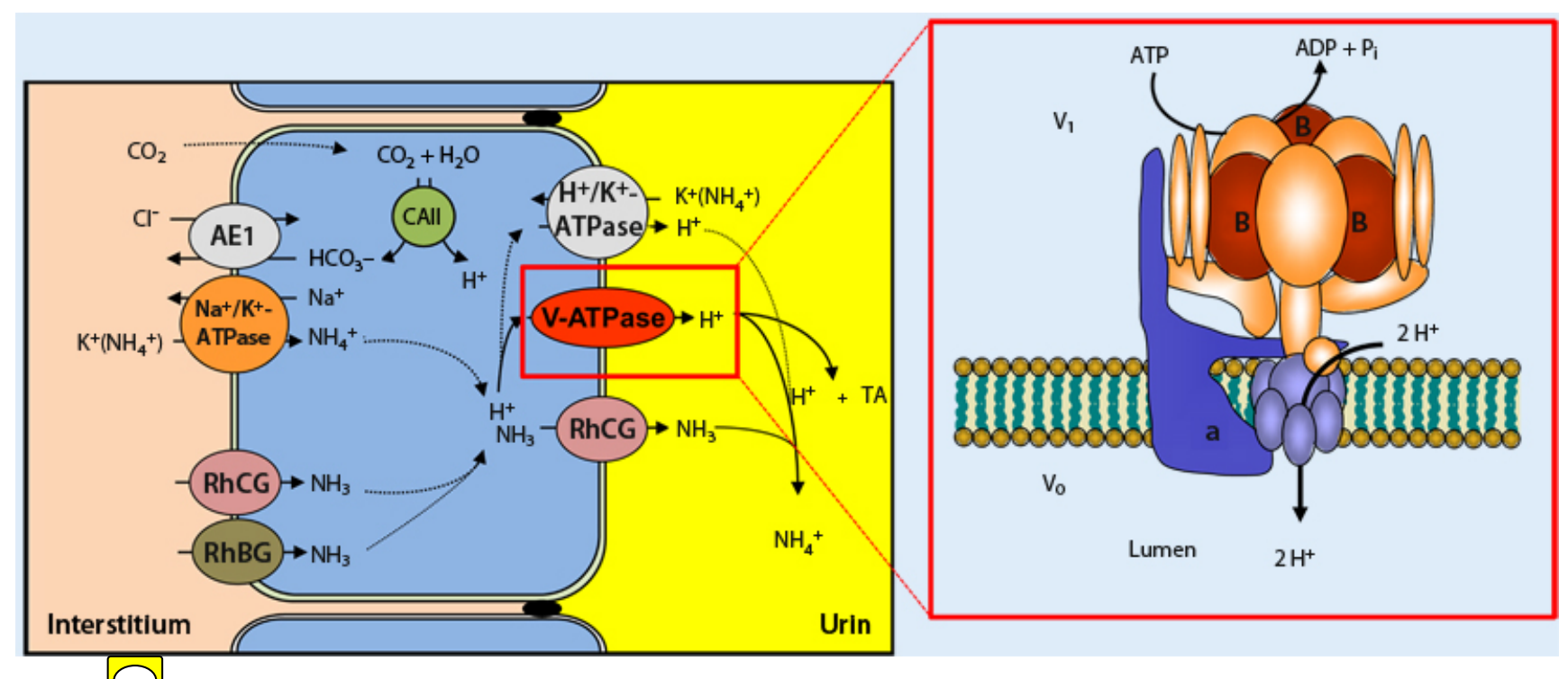

Abb. $2 \Omega$ hematische Darstellung der Typ-A-Schaltzelle im distalen Nephron und der vakuolären

Protonenpumpe: Die rot/orange markierten Anteile der Pumpe entsprechen dem V1-Anteil, die blau gehaltenen Anteile dem V0-Sektor; die B- und a-Untereinheiten sind markiert und kommen in verschiedenen Isoformen vor; B1- und a4-Isoform können bei Patienten mit distaler renaler tubulärer Azidose (dRTA) und Nephrokalzinose mutiert sein ( $A E 1$, anion exchanger“ 1, RhCG ,rhesus blood group family type $C$ glycoprotein“, $R h B G$,rhesus blood group family type B glycoprotein“, $C A I I$ Karboanhydrase Typ II, V-ATPase Va (a) en-ATPase)

Die endgültige Urinansäuerung und somit Feinregulierung des Säure-Basen-Haushalts findet in den Sammelrohren statt []ㅡ. Das Sammelrohrsystem beinhaltet das Verbindungssegment, die kortikalen Sammelrohre sowie die äußeren und inneren medullären Sammelrohre. Die Ausscheidung von Protonen und Ammonium findet eng gekoppelt an den oben erwähnten Prozess in den Typ-A-Schaltzellen statt. Dabei werden auf der luminalen Seite Protonen durch $\mathrm{H}^{+}$-ATPase-Protonenpumpen in den Urin sezerniert, während 
Bikarbonat auf der basolateralen Seite ins Blut abgegeben wird (Abb. 2). Entsprechend verursachen Mutationen verschiedener Untereinheiten der Protonenpumpe eine dRTA beim Menschen [6, 7]. Die Sekretion von Protonen in den Urin führt zu einer Ansäuerung des Urins mit einem minimalen $\mathrm{pH}$ von etwa 4,5-5. Eine weitere Ansäuerung ist aufgrund der limitierten Protonensekretion durch die Protonenpumpen gegen einen Protonengradienten von mehr als 3,5 pH-Einheiten (intrazellulärer $\mathrm{pH}$ von ca. 7,2 vs. maximaler Urin-pH von ca. 4,5) nicht möglich. Ein Liter eines Urins mit einem pH von 4,5 würde jedoch ungepuffert nur etwa 30 umol Protonen enthalten, was von der auszuscheidenden Menge von etwa 40 mmol Protonen weit entfernt wäre, sodass weitere Mechanismen notwendig sind, um die Menge an Protonen im Urin ausreichend zu neutralisieren und damit die maximale Protonenausscheidung zu steigern. Dies geschieht über Pufferung der Protonen durch Phosphat, Zitrat und andere Puffer, die gemeinsam als sog. titrierbare Säuren (auch fixe oder nichtvolatile Säuren genannt) bezeichnet werden. Diese Anionen besitzen die Fähigkeit, Protonen zu binden und wirken somit als Basen, aufgrund der „historischen“ Analysemethode werden sie jedoch als titrierbare Säuren bezeichnet. Des Weiteren wird im Sammelrohr eine große Menge an Ammoniak ausgeschieden, welches nach Bindung von Protonen als Ammonium $\left(\mathrm{NH}_{4}^{+}\right)$ausgeschieden wird. Die Summe von Ammonium und titrierbaren Säuren minus Bikarbonat (bei saurem Urin-pH vernachlässigbar) im Urin wird als Nettosäureausscheidung bezeichnet []]. Das Gas $\mathrm{NH}_{3}$ kann relativ leicht durch die Zellmembran vieler Gewebe diffundieren, in der Niere sind allerdings mehrere Nephronabschnitte (u. a. der dicke aufsteigende Ast der Henle-Schleife und das Sammelrohr) für $\mathrm{NH}_{3}$ undurchlässig. Kürzlich erschienene Arbeiten haben jedoch gezeigt, dass alle Subtypen von Schaltzellen RhCG (,rhesus blood group family type C glycoprotein“, ein Protein aus der Rhesusproteinfamilie) exprimieren, welche als Ammoniakgaskanäle funktionieren und damit die Ausscheidung von $\mathrm{NH}_{3}$ in den Urin ermöglichen (Abb. 2; [9] ). Entsprechend ist die Expression bzw. die Aktivität von RhCG bei Azidose parallel zur stimulierten Ammoniagenese gesteigert [10].

Neben Typ-A-Schaltzellen kommen Typ-B-Schaltzellen entlang des späten distalen Konvoluts, des Verbindungssegments und des kortikalen Sammelrohrs vor. Diese Zellen exprimieren einen apikal gelegenen Bikarbonat-Chlorid-Austauscher (Pendrin/SLC26A4), wodurch sie im Austausch mit Chlorid Bikarbonat in den Urin sezernieren können [11]. Expression und Aktivität von Pendrin sind entsprechend bei Azidose herunterreguliert und 
bei Alkalose stimuliert $[\underline{12}, \underline{13}]$. Des Weiteren spielen Pendrin und somit Typ-B-Schaltzellen aufgrund der Fähigkeit zur Chloridrückresorption eine wichtige Rolle bei der Blutdruckregulation. Pendrin wird durch Aldosteron und Angiotensin stimuliert, und pendrindefiziente Mäuse sind resistent gegen Salz- bzw. aldosteroninduzierte Hypertonie [14, $\underline{15}, \underline{16}]$.

\section{Fallvignette einer angeborenen distalen renal-tubulären Azidose}

Ein 35-jähriger Patient wurde von den Kollegen der Urologie zur Metaphylaxe bei rezidivierender Nephrolithiasis und vorbestehender Nephrokalzinose zugewiesen. Anamnestisch wurde bereits im Alter von 6 Wochen bei massiver metabolischer Azidose (Blut-pH: 6,98, Bikarbonat: 3,3 mmol/l) mit einem alkalischen Urin-pH (Urin-pH: 7,0) im Kinderspital Zürich die Diagnose einer distalen renal-tubulären Azidose gestellt. Radiologisch war bereits damals eine Nephrokalzinose erkennbar. Bei der Stammbaumanalyse bestand der Verdacht auf eine autosomal-rezessive Erkrankung. Eine Alkalitherapie mit einer Kombination von Kaliumzitrat und Natriumbikarbonat wurde begonnen. Dennoch kam es im weiteren Verlauf insbesondere im jungen Erwachsenenalter aufgrund von Malcompliance zu häufigen Koliken sowie rezidivierender Steinbildung (100\% Apatit) und Steinabgängen, die einerseits aufgrund der Koliken zu einer Opiatabhängigkeit und andererseits aufgrund der häufigen Steinabgänge mit Infekten zu einer Niereninsuffizienz geführt haben. Des Weiteren entwickelte der Patient im Verlauf eine Innenohrschwerhörigkeit, welche mit einem Hörgerät versorgt wurde. Bei der Vorstellung in unserer Klinik bestand eine Nephrokalzinose mit einer chronischen Niereninsuffizienz, entsprechend KDIGO-Stadium G3a-b (Abb. 3). Weiterhin lagen eine Hypokaliämie (Serumkalium: 3,1 mmol/1) und eine metabolische Azidose mit einem Serumbikarbonat von 15 mmol/1 vor. Auch zu diesem Zeitpunkt bestand der Verdacht auf eine mäßige Compliance bezüglich der Medikamenteneinnahme. Die Knochendichteuntersuchung zeigte eine Osteopenie bei normalen Serumkalzium-, 25- und 1,25-Dihydroxyvitamin-D- und Parathormonwerten. Serumphosphat war mit 0,67 mmol/1 leicht erniedrigt. Eine Osteopenie oder eine manifeste Osteoporose kommt aufgrund der Hyperkalziurie bei Patienten mit angeborener dRTA häufig vor. Eine genetische Analyse im Département de Génétique am Hôpital Européen Georges Pompidou in Paris ergab eine homozygote Mutation (p.Gln753*) im ATP6V0A4-Gen (a4-Untereinheit der 
Protonenpumpe). Trotz der Therapie mit Kaliumzitrat berichtet der Patient über rezidivierende Spontansteinabgänge, wobei der hochgradige Verdacht besteht, dass die Medikation nicht regelmäßig eingenommen wird. Erfreulicherweise hat sich die chronische Niereninsuffizienz in den letzten 3 Jahren nicht verschlechtert.
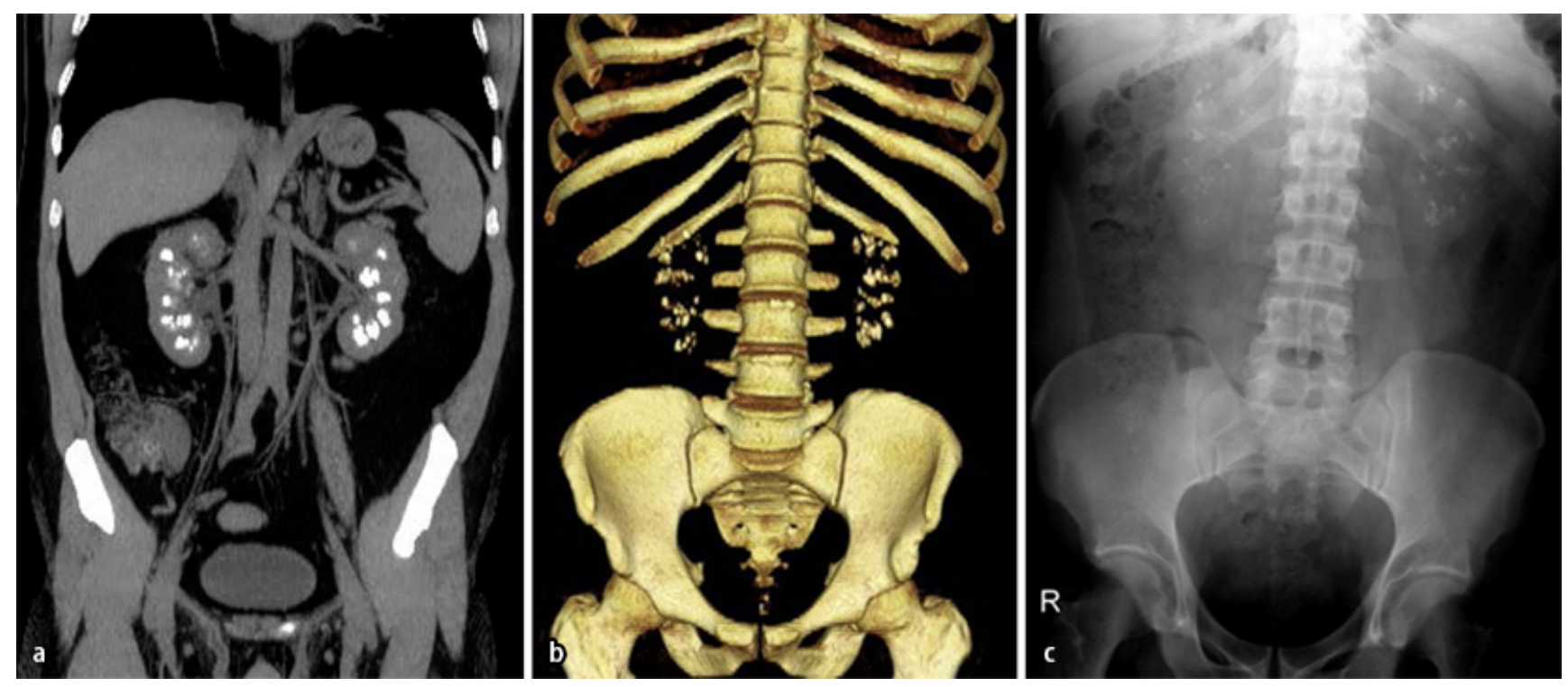

Abb. 3 Medulläre Nephrokalzinose: Sowohl in der Computertomographie (links, Mitte) als auch in der Abdomenübersichtsaufnahme (rechts) zeigt sich die medulläre Nephrokalzinose beider Nieren als typisches Merkmal bei Patienten mit angeborener distaler renal-tubulärer Azidose

\section{Angeborene Formen der distalen renal-tubulären Azidose}

Hereditäre Störungen des Säure-Basen-Haushalts sind selten und werden verursacht durch

1. Mutationen in einer Reihe von Transportproteinen und Enzymen,

2. Defekte in Komponenten des Renin-Angiotensin-Aldosteron Systems,

3. schwere Malformationen der Niere.

Bei den renal-tubulären Azidosen handelt es sich um tubuläre Erkrankungen, bei denen der Defekt in spezifischen Tubulussegmenten lokalisiert ist, welche gleichzeitig auch der Nomenklatur bzw. der Klassifikation zugrunde liegen. Bei der dRTA (auch klassische oder Typ-1-dRTA genannt) handelt es sich um eine primäre Störung der Urinansäuerung in den Sammelrohren (Urin-pH >5,3), welche konsekutiv zu einer inadäquaten Ausscheidung von Ammonium und titrierbaren Säuren führt [1]. Die Folge ist eine hyperchlorämische metabolische Azidose (mit normaler Anionenlücke), deren Komplikationen entsprechend 
für den Phänotyp in Form von Hypokaliämie, Wachstumsretardierung im Kindesalter, Osteopenie/Osteoporose sowie Nephrolithiasis bzw. Nephrokalzinose verantwortlich sind. Des Weiteren besteht bei einigen Patienten eine Polyurie, welche vermutlich teilweise durch die Hypokaliämie und sekundäre Störungen in den Hauptzellen bedingt ist [17, 18]. Die inkomplette Form der dRTA ist ebenfalls durch eine inadäquate Urinansäuerung gekennzeichnet, allerdings präsentieren die Patienten unter Alltagsbedingungen einen normalen Blut-pH und normale Plasmabikarbonatspiegel. Der Defekt wird somit erst nach einem Säurebelastungstest mit Gabe von Ammoniumchlorid ersichtlich, wo sich ein ,alkalischer“ Urin-pH größer 5,3 zeigt [19].

\section{$>$ Neben den bekannten kommen weitere unbekannte Gene als Ursache einer dRTA in Frage}

Bisher sind Mutationen in 3 verschiedenen Transportproteinen, im Chlorid-Bikarbonat-Austauscher AE1/SLC4A1 oder in den B1/ATP6V1B1-bzw. a4/ATP6V0A4-Untereinheiten der Protonenpumpe beschrieben, welche eine dRTA verursachen können. Interessanterweise können jedoch nicht alle Fälle von angeborener Typ-1-dRTA durch Mutationen in diesen Genen erklärt werden, sodass weitere unbekannte Gene als Ursache einer dRTA in Frage kommen. AE1-Mutationen werden vor allem autosomal-dominant vererbt, während Protonenpumpendefekte autosomal-rezessiven Erbgängen folgen. Ausnahmen sind seltene Fälle von Patienten mit 2 mutierten Allelen für SLC4A1 und Hinweise, dass ein defektes Allel bei ATP6V1B1 genügt, um eine inkomplette dRTA zu verursachen [20]. Die Protonenpumpe besteht aus einem Komplex, der sich aus

2 Domänen zusammensetzt:

- zytosolische katalytische $\mathrm{V}_{1}$-Domäne, welche ATP hydrolysiert (mit den 8 Untereinheiten $\mathrm{A}-\mathrm{H})$;

- membrangebundene $\mathrm{V}_{0}$-Domäne, die für den Protontransfer zuständig ist (mit den Untereinheiten a, c, c“", d, e; [21] $)$.

Die B1-Untereinheit ist Teil der $\mathrm{V}_{1}$-Domäne, während die a4-Untereinheit zur $\mathrm{V}_{0}$-Domäne gehört (Abb. 2).

Die B1-Untereinheit wird entlang des Nephrons nicht nur in Schaltzellen, sondern auch im aufsteigenden Ast der Henle-Schleife exprimiert. Die a4-Untereinheit wird hingegen 
neben den Schaltzellen auch in proximalen Tubuluszellen sowie in der Henle-Schleife gefunden [22]. Des Weiteren werden beide Untereinheiten in extrarenalen Organen wie z. B. im Nebenhoden (Epididymis) oder in der Stria vascularis des Innenohrs exprimiert. Interessanterweise scheint dabei die Mutation bezüglich Fertilität keine Rolle zu spielen, wohingegen insbesondere Patienten mit einer B1-Mutation von einer sensorineuralen Schwerhörigkeit betroffen sind, welche im Gegensatz zur metabolischen Azidose nicht auf eine Alkalitherapie anspricht [] ]. Patienten mit einer a4-Mutation können ebenfalls eine sensorineurale Schwerhörigkeit entwickeln, welche sich typischerweise ineinem im Vergleich zu B1-Patienten etwas späteren Lebensalter manifestiert [23] . Bei einzelnen Patienten mit B1-Mutation besteht eine Schwindelsymptomatik, welche durch ein erweitertes vestibuläres Aquäduktsyndrom verursacht ist, wobei die genaue Pathophysiologie noch nicht geklärt ist [24].

Die meisten bisher beschriebenen Mutationen der B1-Untereinheit führen entweder zu einer fehlerhaften Anordnung bzw. Zusammensetzung oder zur Dysfunktion der $\mathrm{H}^{+}$-ATPase-Protonenpumpe. Diese Befunde konnten anhand verschiedener Experimente sowohl in Zellsystemen als auch in B1-defizienten Mäusen bestätigt werden [25]. Entsprechend zeigen B1-defiziente Mäuse nach einer Säurebelastung im Sinne eines Ammoniumchloridbelastungstests das typische Bild einer renal-tubulären Azidose mit einem alkalischen Urin als Zeichen der inadäquaten Ansäuerung des Urins. Die genauere pathophysiologische Bedeutung der a4-Untereinheit wurde erst durch kürzlich publizierte Experimente an a4-defizienten Mäusen geklärt [26, 27]. Entsprechend B1-defizienten Mäusen und dem klinischen Phänotyp beim Menschen zeigen die a4-defizienten Tiere eine schwere metabolische Azidose mit Hypokaliämie und Nephrokalzinose sowie eine verminderte Knochendichte. Des Weiteren bestehen in diesem Mausmodell eine ausgeprägte Schwerhörigkeit sowie eine Störung des Geruchssinns. Da die a4-Untereinheit auch im proximalen Tubulus exprimiert ist und Protonenpumpen dort eine wichtige Rolle bei der rezeptorvermittelten Resorption von niedermolekularen Proteinen spielen, zeigen Mäuse mit a4-Deletion sowie Patienten mit a4-Mutation eine vermehrte Ausscheidung von niedermolekularen Proteinen [27]. Ob dies auch ein Grund dafür ist, dass Patienten mit a4-Mutation wie in unserer Fallvignette ein höheres Risiko für eine Niereninsuffizienz haben, ist jedoch noch ungeklärt. 
Neben Typ-A-Schaltzellen wird AE1 auch in Erythrozyten exprimiert. Abgesehen von der autosomal-dominanten Form wurden im südostasiatischen Raum selten autosomal-rezessive Fälle beschrieben, die eine Sphärozytose, die südostasiatische Ovalozytose oder eine dRTA verursachen können [1]. Interessanterweise kommt es bei der klinischen Manifestation jedoch meistens entweder zu einem renalen oder zu einem hämatologischen Erscheinungsbild und nur sehr selten zu einem kombinierten Krankheitsbild. Die häufigste rezessiv vererbte AE1-Mutation (G701D) führt zu einer dRTA ohne hämatologischen Phänotyp. Dies ist vermutlich durch die Interaktion von AE1 mit dem Chaperon-Protein Glycophorin bedingt, welches die Dysfunktion des mutierten Proteins in Erythrozyten verhindert. Glycophorin ist allerdings nicht in Schaltzellen exprimiert, was somit den renalen Phänotyp dieser Mutation erklärt. Eine Reihe weiterer Mutationen wurde bisher beschrieben und charakterisiert, welche gehäuft in Südostasien vorkommen und eher mit dem hämatologischen Phänotyp assoziiert sind. Im Gegensatz zu den rezessiven AE1-Mutationen kommt die dominante Form häufiger bei Kaukasiern vor und verursacht nur äußerst selten einen hämatologischen Phänotyp. Diese Mutationen können jeweils zu einer fehlerhaften Lokalisation von AE1 an der basolateralen Membran von Typ-A-Schaltzellen oder zu einer Störung des Transports des Proteins zur Zellmembran sowie zu einem Aktivitätsverlust führen. Entsprechend zeigen AE1-defiziente Mäuse im Vergleich zu den heterozygoten Tieren einen schweren Phänotyp mit hoher postnataler Mortalität und eine ausgeprägte hämolytische Anämie [28]. Weiterhin zeigen die überlebenden Tiere eine dRTA mit schwerer hyperchlorämischer metabolischer Azidose, Wachstumsretardierung, Nephrokalzinose und niedriger Zitratausscheidung im Urin. Zusätzlich besteht eine Exsikkose aufgrund eines vermutlich renal bedingten Konzentrationsdefekts infolge von gestörter Aquaporin-2-Regulation.

\section{Angeborene und erworbene dRTA als Differenzialdiagnose bei Nephrokalzinose oder rezidivierender Nephrolithiasis im Erwachsenenalter}

Die Differenzialdiagnose der Nephrokalzinose ist vielfältig und beinhaltet eine große Zahl an Erkrankungen mit sehr unterschiedlicher Pathophysiologie wie z. B. primäre Hyperoxalurie, Sarkoidose, Markschwammnieren, primärer Hyperparathyreoidismus sowie dRTA und weitere (Tab. 1). Da jedoch eine Nephrokalzinose unbehandelt zu einer 
chronischen Niereninsuffizienz führen kann, ist eine frühzeitige und richtige Diagnosestellung essenziell. Eine Korrektur der Azidose im Rahmen einer dRTA kann somit sowohl eine Wachstumsstörung bei den Kindern als auch eine weitere Progression der Nephrokalzinose verhindern und somit zu einem Erhalt der Nierenfunktion beitragen.

Eine komplette dRTA wird typischerweise aufgrund der Schwere der Symptomatik (Erbrechen, Gedeihstörung, Nephrokalzinose, Osteomalazie) bereits im Kindesalter diagnostiziert. Allerdings können sich einzelne Patienten mit milden klinischen Symptomen wie einer milderen metabolischen Azidose, Hypozitraturie, einem Zufallsbefund von Nierensteinen oder renaler Kalzifikation präsentieren [29]. Daher ist die dRTA eine wichtige Differenzialdiagnose bei Nephrokalzinose oder rezidivierender Nephrolithiasis auch im Erwachsenenalter. Insbesondere die autosomal-dominante Form der angeborenen dRTA (AE1-Mutation) kann erst im jungen Erwachsenenalter manifest werden [4]. Bei der Differenzialdiagnose ist auch die Kenntnis der Steinzusammensetzung - im Falle von angeborener dRTA z. B. 100\% Apatit - sehr hilfreich. Des Weiteren sollte eine gezielte Anamnese bezüglich Hörleiden oder Schwindelsymptomatik erfolgen, da die meisten Patienten bereits mit einem Hörgerät versorgt worden sind und somit bei Erstkontakt im Erwachsenenalter diesbezüglich unauffällig erscheinen. Ebenfalls sind eine ausführliche Familienanamnese und evtl. eine Familienberatung wie bei allen genetischen Erkrankungen notwendig.

\section{Fazit für die Praxis}

- Die Niere spielt bei der Regulation und Aufrechterhaltung des Säure-Basen-Haushalts eine wichtige Rolle. Die Bedeutung dieser Aufgabe zeigt sich vor allem durch die verschiedenen Formen von renalen Azidosen, die bei angeborenen oder erworbenen Störungen der renalen Säureausscheidung auftreten können.

- Bei der distalen renal-tubulären Azidose (dRTA) handelt es sich um eine Störung der Urinansäuerung. Die Folge ist eine hyperchlorämische metabolische Azidose mit einem alkalischen Urin-pH. Die Schwere der klinischen Symptome kann je nach Mutation variieren.

- Bei der Mehrzahl der Patienten tritt zusätzlich eine Innenohrschwerhörigkeit auf.

- Die Erkrankung kann im frühen Kindesalter als auch im jungen Erwachsenenalter auftreten und stellt eine wichtige Differenzialdiagnose bei Nephrokalzinose oder rezidivierender 
Nephrolithiasis dar, insbesondere falls es sich bei der Steinzusammensetzung um 100\% Apatit handelt oder die Patienten gleichzeitig an einer Schwerhörigkeit leiden.

Einhaltung ethischer Richtlinien Interessenkonflikt. N. Mohebbi und C.A. Wagner geben an, dass kein Interessenkonflikt besteht.

Dieser Beitrag beinhaltet keine Studien an Menschen oder Tieren.

Danksagung Wir danken Rosa Vargas-Poussou für die Durchführung der genetischen Analyse.

\section{Literatur}

1. Wagner CA, Devuyst O, Bourgeois S, Mohebbi N (2009) Regulated acid-base transport in the collecting duct. Pflugers Arch 458(1):137-156

2. Biner HL, Arpin-Bott MP, Loffing J et al (2002) Human cortical distal nephron: distribution of electrolyte and water transport pathways. J Am Soc Nephrol 13(4):836-847

3. Alper SL Natale J, Gluck S et al (1989) Subtypes of intercalated cells in rat kidney collecting duct defined by antibodies against erythroid band 3 and renal vacuolar H+-ATPase. Proc Natl Acad Sci U S A 86(14):5429-5433

4. Karet FE, Gainza FJ, Györy AZ et al (1998) Mutations in the chloride-bicarbonate exchanger gene AE1 cause autosomal dominant but not autosomal recessive distal renal tubular acidosis. Proc Natl Acad Sci U S A 95(11):6337-6342

5. Gottschalk CW, Lassiter WE, Mylle M (1960) Localization of urine acidification in the mammalian kidney. Am J Physiol 198:581-585

6. Karet FE, Finberg KE, Nelson RD et al (1999) Mutations in the gene encoding B1 subunit of H+-ATPase cause renal tubular acidosis with sensorineural deafness. Nat Genet 21(1):84-90

7. Karet FE, Finberg KE, Nayir A et al (1999) Localization of a gene for autosomal recessive distal renal tubular acidosis with normal hearing (rdRTA2) to 7q33-34. Am J Hum Genet 65(6):1656-1665

8. Hamm LL, Simon EE (1987) Roles and mechanisms of urinary buffer excretion. Am J Physiol 253(4 Pt 2):F595-F605 
9. Biver S, Belge H, Bourgeois S et al (2008) A role for Rhesus factor Rhcg in renal ammonium excretion and male fertility. Nature 456(7220):339-343

10. Bourgeois S, Bounoure L, Christensen EI et al (2013) Haploinsufficiency of the ammonia transporter Rhcg predisposes to chronic acidosis: Rhcg is critical for apical and basolateral ammonia transport in the mouse collecting duct. J Biol Chem 288(8):5518-5529

11. Kim YH, Kwon TH, Frische S et al (2002) Immunocytochemical localization of pendrin in intercalated cell subtypes in rat and mouse kidney. Am J Physiol Renal Physiol 283(4):F744-F754

12. Hafner P, Grimaldi R, Capuano P et al (2008) Pendrin in the mouse kidney is primarily regulated by $\mathrm{Cl}$ - excretion but also by systemic metabolic acidosis. Am J Physiol Cell Physiol 295(6):C1658-C1667

13. Frische S, Kwon TH, Frøkiaer J et al (2003) Regulated expression of pendrin in rat kidney in response to chronic $\mathrm{NH} 4 \mathrm{Cl}$ or NaHCO3 loading. Am J Physiol Renal Physiol 284(3):F584-F593

14. Pech V, Kim YH, Weinstein AM et al (2007) Angiotensin II increases chloride absorption in the cortical collecting duct in mice through a pendrin-dependent mechanism. Am J Physiol Renal Physiol 292(3):F914-F920

15. Verlander JW, Hassell KA, Royaux IE et al (2003) Deoxycorticosterone upregulates PDS (Slc26a4) in mouse kidney: role of pendrin in mineralocorticoid-induced hypertension. Hypertension 42(3):356-362

16. Wagner CA, Mohebbi N, Capasso G, Geibel JP (2011) The anion exchanger pendrin (SLC26A4) and renal acid-base homeostasis. Cell Physiol Biochem 28(3):497-504

17. Gueutin V, Vallet M, Jayat M et al (2013) Renal $\beta$-intercalated cells maintain body fluid and electrolyte balance. J Clin Invest 123(10):4219-4231

18. Sebastian A, McSherry E, Morris RC Jr (1976) Impaired renal conservation of sodium and chloride during sustained correction of systemic acidosis in patients with type 1, classic renal tubular acidosis. J Clin Invest 58(2):454-469

19. Wrong O, Davies HE (1959) The excretion of acid in renal disease. Q J Med 28(110):259-313 
20. Zhang J, Fuster DG, Cameron MA et al (2014) Incomplete distal renal tubular acidosis from a heterozygous mutation of the V-ATPase B1 subunit. Am J Physiol Renal Physiol 307(9):F1063-F1071

21. Wagner CA, Finberg KE, Breton S et al (2004) Renal vacuolar H+-ATPase. Physiol Rev 84(4):1263-1314

22. Stehberger PA, Schulz N, Finberg KE et al (2003) Localization and regulation of the ATP6V0A4 (a4) vacuolar H+-ATPase subunit defective in an inherited form of distal renal tubular acidosis. J Am Soc Nephrol 14(12):3027-3038

23. Stover EH, Borthwick KJ, Bavalia C et al (2002) Novel ATP6V1B1 and ATP6V0A4 mutations in autosomal recessive distal renal tubular acidosis with new evidence for hearing loss. J Med Genet 39(11):796-803

24. Mohebbi N, Vargas-Poussou R, Hegemann SC et al (2013) Homozygous and compound heterozygous mutations in the ATP6V1B1 gene in patients with renal tubular acidosis and sensorineural hearing loss. Clin Genet 83(3):274-278

25. Finberg KE, Wagner CA, Bailey MA et al (2005) The B1-subunit of the $\mathrm{H}(+)$ ATPase is required for maximal urinary acidification. Proc Natl Acad Sci U S A 102(38):13616-13621

26. Lorente-Canovas B, Ingham N, Norgett EE et al (2013) Mice deficient in H+-ATPase a4 subunit have severe hearing impairment associated with enlarged endolymphatic compartments within the inner ear. Dis Model Mech 6(2):434-442

27. Hennings JC, Picard N, Huebner AK et al (2012) A mouse model for distal renal tubular acidosis reveals a previously unrecognized role of the V-ATPase a4 subunit in the proximal tubule. EMBO Mol Med 4(10):1057-1071

28. Stehberger PA, Shmukler BE, Stuart-Tilley AK et al (2007) Distal renal tubular acidosis in mice lacking the AE1 (band3) Cl-/HCO3- exchanger (slc4a1). J Am Soc Nephrol 18(5):1408-1418

29. Karet FE (2002) Inherited distal renal tubular acidosis. J Am Soc Nephrol 13(8):2178-2184 
Tab. 1 Differenzialdiagnosen der Nephrokalzinose

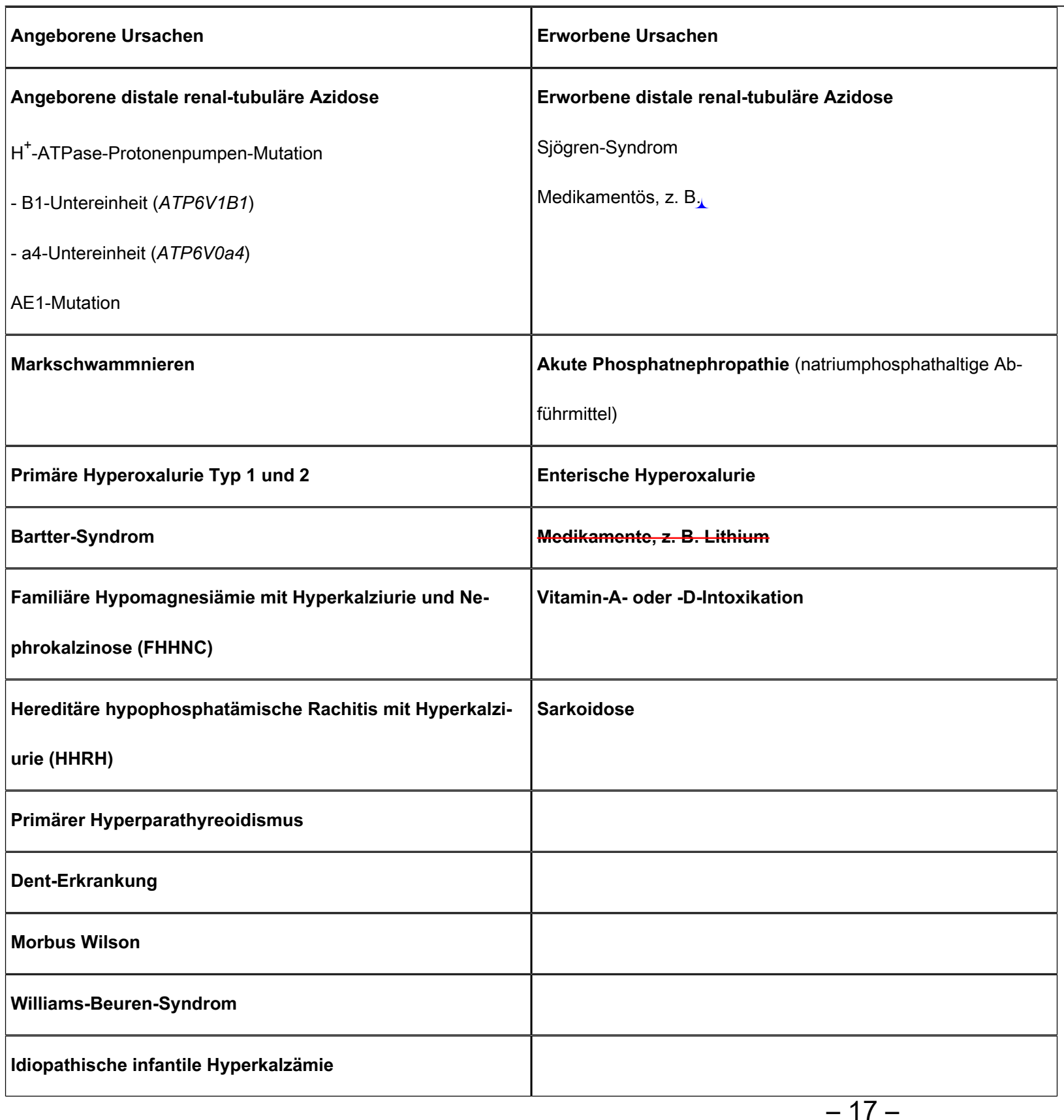


Erworbene Ursachen

Zahnschmelz-Nieren-Syndrom („,enamel renal syndrome“) 